\title{
Florence Fix, Barbe-Bleue et l'esthétique du secret de Charles Perrault à Amélie Nothomb
}

\section{Roberta Sapino}

\section{(2) OpenEdition}

1 Journals

\section{Edizione digitale}

URL: http://journals.openedition.org/studifrancesi/1676

DOI: 10.4000/studifrancesi. 1676

ISSN: 2421-5856

Editore

Rosenberg \& Sellier

\section{Edizione cartacea}

Data di pubblicazione: 1 novembre 2014

Paginazione: 652-653

ISSN: 0039-2944

\section{Notizia bibliografica digitale}

Roberta Sapino, «Florence Fix, Barbe-Bleue et l'esthétique du secret de Charles Perrault à Amélie Nothomb », Studi Francesi [Online], 174 (LVIII | III) | 2014, online dal 01 novembre 2014, consultato il 18 septembre 2020. URL : http://journals.openedition.org/studifrancesi/1676 ; DOI : https://doi.org/ 10.4000/studifrancesi. 1676

Questo documento è stato generato automaticamente il 18 settembre 2020 .

\section{(c)}

Studi Francesi è distribuita con Licenza Creative Commons Attribuzione - Non commerciale - Non opere derivate 4.0 Internazionale. 


\title{
Florence Fix, Barbe-Bleue et l'esthétique du secret de Charles Perrault à Amélie Nothomb
}

\author{
Roberta Sapino
}

\section{NOTIZIA}

FLORENCE FIX, Barbe-Bleue et l'esthétique du secret de Charles Perrault à Amélie Nothomb, Paris, Hermann, 2014, pp. 233.

1 Chi è Barbablù, e perché non smette di affascinarci? - si chiede Florence FIX, docente presso l'Université de la Lorraine e già autrice di un testo sul personaggio di Medea. Perché il mito dell'uomo dall'aspetto incongruo e dalla vita inquietante è innanzitutto un racconto della curiosità: curiosità delle giovani donne che gli si avvicinano, del popolo vociferante, ma anche curiosità del lettore, desideroso di scoprire cosa si nasconda oltre la porta proibita e di capire se dietro la barba del personaggio non si celi forse un essere storico in carne ed ossa.

2 Numerose ipotesi sono state avanzate a questo proposito: se volessimo individuare delle ispirazioni storiche, afferma l'A. nell'introduzione (Variations autour du bleu, pp. 7-22), dovremmo ricercarle nelle controverse vicende matrimoniali di Enrico VIII, o in quelle di Conomor conte di Poher, che - leggenda vuole - uccideva le sue mogli non appena esse rimanevano incinte, o ancora nello scandalo di Gilles de Rais, compagno di Giovanna d'Arco condannato a morte per aver torturato e ucciso svariati giovani e bambini. Ma Barbablù è innanzitutto il protagonista del conte omonimo che Perrault pubblicò nel 1697, e che da allora è stato oggetto di numerosissime riscritture.

3 Scopo del volume è proprio addentrarsi in questa costellazione di riprese e reinterpretazioni per rilevare linee di tendenza e particolarità individuali, in un'impresa quasi archeologica di analisi, confronto e ricerca di senso. Il corpus è voluminoso: una ventina di testi, tra romanzi, racconti, opere teatrali, di area francese 
(Bruno Castan, Alphonse Daudet, Pierrette Fleutiaux, Anatole France, Carole Fréchette, Nicolas Fretel, Claire Legendre, Maurice Maeterlinck, Amélie Nothomb, Jean-Michel Rabeux, Henri de Régnier, Alfred Savoir, Henri-Jean Gauthier-Villars - più noto come Willy, il primo marito della scrittrice Colette - oltre, evidentemente, a Charles Perrault) e europea (Margaret Atwood, Béla Bartók, Angela Carter, Charles Dickens, Max Frisch, Maria-Luisa Linarès, Dea Loher, George Steiner), con sporadiche incursioni in ambito cinematografico; al suo interno Florence Fix si muove seguendo tre grandi assi tematici. Il primo capitolo, intitolato Figures de l'impossible (pp. 23-90), introduce la figura di Barbablù considerandola non come entità singola, ma come polo all'interno di una relazione coniugale nata sotto il segno della discordanza e dell'interdit. Cosa conduce una giovane donna di origini modeste a legarsi a un uomo ricchissimo, più avanti negli anni, segnato da un inquietante marchio fisico? Senza dubbio il conte di Perrault porta in sé l'eco dell'ingresso della borghesia nella società francese del diciassettesimo secolo, afferma Fix, e tuttavia le varie riscritture lasciano emergere un altro punto fondamentale nella relazione: in virtù del suo aspetto mostruoso, talvolta grottesco, l'uomo dalla barba blu sfida la nozione stessa di identità, suscitando nelle vittime designate, generalmente caratterizzate da una qualche mancanza, una forma di seduzione rivolta non alla persona, ma alla vertigine dell'impossibile che essa rappresenta. Interrogazione identitaria non implica, sottolinea l'autrice, evoluzione: al contrario, quello di Barbablù è un conte dell'immobilità, del tempo sospeso, della rigidità del terrore. A partire dai cadaveri delle donne rinchiuse nella stanza proibita (che in Amélie Nothomb sono, significativamente, sostituite da fotografie di altissima qualità e durata), misteriosamente sottratti al processo di decomposizione, nulla si muove nella reggia, e anche i personaggi - a differenza di altre storie della mostruosità, come La Bella e la Bestia - mantengono intatte e immutate le loro caratteristiche iniziali. Il silenzio dell'inavouable e la tendenza alla ripetizione, evidente nella macabra collezione che, osserva Fix, sottrae l'oggetto al valore d'uso per elevarlo a un livello assoluto di pura memoria, diventano allora cifra di un personaggio la cui ricerca di completezza si scontra irrimediabilmente con il principio di realtà.

Il secondo capitolo, La curiosité est un vilain défaut (pp. 91-148) riprende le riflessioni avanzate in precedenza per mostrare come la vicenda di Barbablù, a prescindere dalla riscrittura, sia pervasa da un'interrogazione sullo sguardo: audacia del voler vedere (o non vedere) a tutti i costi, ma anche difficoltà a discernere e investire di senso ciò cui si è assistito. Segnalando la superficialità di quella lettura che fa del testo un semplice mito della curiosità femminile punita, Fix propone piste di ricerca volte innanzitutto a comprendere l'enigma costituito dall'universo mentale non tanto di Barbablù quanto della sua sposa: cosa si cela dietro al desiderio di vedere l'orrore? Nel conte di Perrault è in gioco l'affermarsi della soggettività moderna, l'insubordinazione che porta all'affermazione di sé, e tuttavia, una volta aperta la porta, la ricerca del sapere si gela in una contemplazione autoreferenziale del proprio terrore e non sa tramutarsi in vera conoscenza del mondo. In questo senso, Barbablù rappresenterebbe un mito non tanto del segreto inaccessibile, quanto della permanenza dell'ignoranza davanti all'inspiegabile.

6 Il terzo capitolo, intitolato Anne, ma soeur Anne... (pp. 149-208) parte proprio da questa riflessione, estendendola in una lettura del mito come percorso di ricerca di senso. La chiave insanguinata, osserva l'autrice, apre alla sposa la voragine dell'assenza di senso, obbligandola a ripensare il proprio essere rispetto al caos dell'inspiegabile. Barbablù, 
dal canto suo, custodisce un segreto che non ha scopo né finché si mantiene nell'ombra, né nel momento in cui viene alla luce. Nell'organizzare una rappresentazione della quale è l'unico spettatore, reiterando a suo piacere il meccanismo di se stesso che guarda la donna che guarda la morte, Barbablù mette lo spettatore-lettore faccia a faccia con l'essenza stessa della violenza: l'inutilità assoluta, l'opacità a qualsiasi spiegazione, il vuoto di senso che ne mantengono intatto e inscalfibile il potere di siderazione.

7 Florence Fix conclude (L'adieu au bleu, pp. 209-218) ripercorrendo le tappe fondamentali della sua analisi: l'uomo mostruoso dalla barba blu non smette di affascinare perché ci mette, reiteratamente, davanti alla necessità di interrogarci sulle questioni dell'identità e della seduzione, sulla volontà di vedere e sull'impossibilità di conoscere, sul nostro rapportarci al tempo e alla memoria; ma anche perché è un mito dell'attesa, della paura che precede la conferma del crimine e che esercita sul lettore quell'attrazione che la stanza proibita esercita sulle spose del récit. 